\title{
ON THE ASYMPTOTIC GROWTH OF SOLUTIONS TO A NONLINEAR EQUATION
}

\author{
S. P. HASTINGS
}

We shall consider the nonlinear integral equation

$$
\dot{x}(t)-\dot{x}(0)+\int_{0}^{t} a(s) g(x(s)) d s=h(t), \quad 0 \leqq t<\infty,
$$

where $a(t)$ and $h(t)$ are continuous for $t \geqq 0$ and $g(x)$ is continuous for all $x$. If $h(t)$ is absolutely continuous on bounded sets, then (1) is equivalent to the differential equation

$$
\ddot{x}(t)+a(t) g(x(t))=h(t),
$$

so our results will hold for solutions of (2). We shall be primarily interested in the nonlinear oscillator, i.e. $a(t)>0, x g(x)>0$ for $x \neq 0$, but in Theorem III below, sign restrictions are removed.

If we consider $h(t)$ to be a sample function of a Brownian motion process $h(t, \omega)$ on a probability space $\Omega$, we see the motivation for considering (1). Equation (2) then represents a nonlinear oscillation driven by "white noise," an illegitimate process in the sense that for almost all $\omega \in \Omega, h(t, \omega)$ is not differentiable.

For an account of the probabilistic aspects of (1) for the case in which $a(t)$ is constant, see [2]. We shall be concerned with bounds on the asymptotic growth of solutions to (1). Various results for the homogeneous case are found in [4], and [5]. Results for the nonhomogeneous case when $a(t)$ is constant are obtained for (1) in [2] and for (2) in [3].

Note that the usual local existence theorems which hold for (2) may be obtained for (1) by considering the equivalent system of equations

$$
\begin{aligned}
\dot{x}(t) & =u(t)+h(t), \\
-\dot{u}(t) & =a(t) g(x(t))
\end{aligned}
$$

as in [2]. Here, of course, $u(t)$ is defined to be $\dot{x}(t)-h(t)$.

The proofs and results of Theorems I and II are essentially the same as those of Waltman in [4] for the homogeneous case, and depend on the well-known lemma due to Gronwall [1, p. 37].

THEOREM I. Suppose $g(x)$ is a monotone nondecreasing odd function

Received by the editors March 18, 1965. 
which is positive for $x>0$ and continuous for all $x$. Suppose also that $a(t)$ is positive and absolutely continuous on bounded sets, and that

$$
\int_{0}^{\infty}\left|a^{\prime}(s)\right| / a(s) d s<\infty .
$$

Let $x(t)$ be a solution to (1) defined at $t=0$. Then $x(t)$ can be extended to $[0, \infty)$, and

$$
x(t)=O\left(t+\int_{0}^{t}|h(s)| d s\right)
$$

as $t$ goes to infinity.

Proof. Condition (3) implies that $a(t)$ is bounded below by some positive number. In fact, the following is obvious:

Lemma I. With $a(t)$ as above, $\sup _{0 \leq t \leq r<\infty}[a(t) / a(\tau)]$ is finite. Further, let $\left\{T_{i}\right\}$ be a nondecreasing sequence of positive numbers and let

$$
\alpha_{i}=\sup _{T_{i} \leqq i \leqq \tau \leqq T_{i+1}}[a(t) / a(\tau)] .
$$

Then $\prod_{i=1}^{\infty} \alpha_{i}<\infty$.

Let $\beta=\sup _{t \geq 0} a(t)^{-1}$. The theorem will follow easily from the next lemma.

Lemma II. Let $[A, B]$ be an interval on which $x(t)$ is defined and such that $\dot{x}(t)-h(t)$ does not change sign on this interval. Let

$$
\begin{aligned}
\alpha_{A, B} & =\sup _{A \leqq t \leqq}[a(t) / a(\tau)], \\
K_{A, B} & =\alpha_{A, B} \exp \left\{\int_{A}^{B}\left|a^{\prime}(s)\right| / a(s) d s\right\}, \\
M_{A, B} & =\int_{A}^{B}|h(s)| d s .
\end{aligned}
$$

Pick $\mu \geqq 0$ so that

$$
\int_{0}^{\mu} g(s) d s=\left(2 \alpha_{A, B}\right)^{-1} \beta(\dot{x}(A)-h(A))^{2} .
$$

Then

$$
|x(B)| \leqq K_{A, B}(|x(A)|+\mu)+\left(2+K_{A, B}\right) M_{A, B} .
$$

Proof of Lemma II. Assume for definiteness that $\dot{x}(t)-h(t)$ is nonnegative on $[A, B]$. Let 


$$
\boldsymbol{H}(t)=\int_{A}^{t} h(s) d s \text { and } y(t)=x(t)-H(t) .
$$

Then

$$
\ddot{y}(t)=-a(t) g(y(t)+H(t)) \leqq-a(t) g\left(y(t)-M_{A, B}\right) .
$$

Therefore, since $\dot{y}(t) \geqq 0$ on $[A, B]$,

$$
\dot{y}(t)^{2} / 2 \leqq \dot{y}(A)^{2} / 2-\int_{A}^{t} a(s) g\left(y(s)-M_{A, B}\right) \dot{y}(s) d s
$$

for $A \leqq t \leqq B$.

Set $G(x)=\int_{0}^{x} g(s) d s$ and note that $G(x)>0$ for $x \neq 0$. Integrating by parts in (4), we have

$$
\begin{aligned}
a(t) G\left(y(t)-M_{A, B}\right) \leqq & \dot{y}(A)^{2} / 2+a(A) G\left(y(A)-M_{A, B}\right) \\
& +\int_{A}^{t} \frac{\left|a^{\prime}(s)\right|}{a(s)} a(s) G\left(y(s)-M_{A, B}\right) d s
\end{aligned}
$$

for $A \leqq t \leqq B$. By Gronwall's inequality,

$$
\begin{aligned}
a(B) G\left(y(B)-M_{A, B}\right) \leqq & {\left[\dot{y}(A)^{2} / 2+a(A) G\left(y(A)-M_{A, B}\right]\right.} \\
& \cdot \exp \left\{\int_{A}^{B}\left|a^{\prime}(s)\right| / a(s) d s\right\} .
\end{aligned}
$$

Recalling that $g(x)$ is an odd, nondecreasing function, we see that

$$
\begin{aligned}
\int_{0}^{\left|y(B)-M_{A}, B\right|} g(s) d s & \leqq K_{A, B}\left[\beta\left(\dot{y}(A)^{2} / 2\right) \alpha_{A, B}^{-1}+\int_{0}^{\left|y(A)-M_{A}, B\right|} g(s) d s\right] \\
& \leqq K_{A, B} \int_{0}^{|y(A)|+M_{A, B}+\boldsymbol{A}} g(s) d s
\end{aligned}
$$

where we use the fact that $\dot{y}(A)=\dot{x}(A)-h(A)$.

Now suppose $p$ and $q$ are positive numbers and $\lambda$ is a number $\geqq 1$. Suppose also that

$$
\int_{0}^{p} g(s) d s \geqq \frac{1}{\lambda} \int_{0}^{q} g(s) d s .
$$

Since $g(x)$ is nondecreasing and positive for $x>0$, it follows that $p \geqq q / \lambda$. From (5), then, we have

$$
|y(B)| \leqq K_{A, B}(|y(A)|+\mu)+\left(1+K_{A, B}\right) M_{A, B} .
$$


Since $|x(t)|=|y(t)+H(t)| \leqq|y(t)|+M_{A, B}$ and $x(A)=y(A)$, the conclusion of Lemma II is immediate.

Now suppose there is a $T \geqq 0$ such that $x(t)$ can be extended past $T$ and such that in the largest interval $[T, N), N \leqq \infty$, to which $x(t)$ can be extended, $\dot{x}(t)-h(t)$ does not change sign. If $N<\infty$, then Lemma II applied to the closed sub-intervals of $[T, N)$ shows that $x(t)$ is bounded for $t<N$. Then (1) implies that $\dot{x}(t)$ is also bounded for $t<N$. But this implies that $x(t)$ can be extended to $[0, N]$, contradicting the definition of $N$. Thus $N=\infty$. Theorem I follows in this case by applying Lemma II to finite intervals $[T, t]$ and letting $t$ go to infinity.

If such a $T$ does not exist, then there must be an unbounded set of zeros of $\dot{x}(t)-h(t)$. In this case we define two disjoint sets $S^{1}$ and $S^{2}$, with $[0, \infty)=S^{1} \cup S^{2}$, as follows:

$S^{1}=\{t \geqq 0 \mid$ There is a neighborhood $(a, b)$ of $t$ in which $\dot{x}(\cdot)-h(\cdot)$ has only a finite number of zeros $\}$.

$S^{2}=\left\{t \geqq 0 \mid t \notin S^{1}\right\}$.

If $t \in S^{2}$, then there is a sequence $t_{i}$ approaching $t$ with $\dot{x}\left(t_{i}\right)-h\left(t_{i}\right)$ $=0$. It follows that $d(\dot{x}(t)-h(t)) / d t=0$ and by $(1), x(t)=0$.

For each $t \in S^{1}$, define $I_{t}$ to be the intersection of $[0, \infty)$ with the union of all neighborhoods of $t$ in which $\dot{x}(\cdot)-h(\cdot)$ has only a finite set of zeros. Supose first that for some $t_{1}, I_{t_{1}}$, is semi-infinite. Then there must exist an increasing and unbounded sequence $\left\{T_{i}\right\}$ with $(-1)^{i}(\dot{x}(t)-h(t)) \geqq 0$ on $\left[T_{i}, T_{i+1}\right], i=1,2, \cdots$.

Let $\gamma_{i}=K_{T_{i}, T_{i+1}}$ and note that $\dot{x}\left(T_{i}\right)=h\left(T_{i}\right)$. Lemma II implies that for $i=1,2, \ldots$

$$
\left|x\left(T_{i+1}\right)\right| \leqq \gamma_{i}\left|x\left(T_{i}\right)\right|+\left(2+\gamma_{i}\right) M_{T_{i}, T_{i+1}} .
$$

Lemma $I$ and condition 3 show that $L=\prod_{i=1}^{\infty} \gamma_{i}$ is finite. It is easy to show by induction that for $n=2,3, \cdots$,

$$
\begin{aligned}
\left|x\left(T_{n}\right)\right| & \leqq\left(\prod_{i=1}^{n-1} \gamma_{i}\right)\left|x\left(T_{1}\right)\right|+3\left(\prod_{i=1}^{n-1} \gamma_{i}\right) \int_{T_{1}}^{T_{n}}|h(s)| d s \\
& \leqq L\left|x\left(T_{1}\right)\right|+3 L \int_{T_{1}}^{T_{n}}|h(s)| d s,
\end{aligned}
$$

and Theorem I follows easily in the same way as when $\dot{x}(t)-h(t)$ does not change sign for $t \geqq T$.

Suppose, on the other hand, that $I_{t}$ is bounded for each $t \in S^{1}$. If, for some $t \in S^{1}, I_{t}=[0, b), b<\infty$, then $[0, t]$ contains only a finite 
number of zeros of $\dot{x}(\cdot)-h(\cdot)$. The argument above shows that

$$
|x(t)| \leqq L_{1}\left(|x(0)|+\left|\mu_{0, t}\right|\right)+3 L_{1} \int_{0}^{t}|h(s)| d s
$$

where

$$
\begin{aligned}
\int_{0}^{\mu_{A, B}} g(s) d s & =\left(2 \alpha_{A, B}\right)^{-1} \beta(\dot{x}(A)-h(A))^{2} \\
L_{1} & =\left[\sup _{0 \leqq \imath \leqq \tau<\infty} a(t) / a(\tau)\right] \exp \left\{\int_{0}^{\infty}\left|a^{\prime}(s)\right| / a(s) d s\right\}
\end{aligned}
$$

and $\alpha_{A, B}$ and $\beta$ are as on p. 41 , for any $B \geqq A \geqq 0$.

Finally, suppose that for some $t, I_{t}=(a, b), a \geqq 0, b<\infty$. Then $a \in S^{2}$ and $x(a)=0$. For any $c$ in $(a, t),[c, t]$ contains only a finite number of zeros of $\dot{x}(\cdot)-h(\cdot)$. Therefore, as before,

$$
|x(t)| \leqq L_{1}\left(|x(c)|+\left|\mu_{c, t}\right|\right)+3 L_{1} \int_{c}^{t}|h(s)| d s .
$$

Letting $c$ approach $a$, we see that

$$
|x(t)| \leqq 3 L_{1} \int_{a}^{t}|h(s)| d s .
$$

We have shown that there is an $M$ such that for any

$$
t \geqq 0, \quad|x(t)| \leqq M+3 L_{1} \int_{0}^{t}|h(s)| d s,
$$

and this completes the proof of Theorem I.

THEOREM II. Suppose $a(t)$ is positive, absolutely continuous on bounded sets, and nondecreasing, and $g(x)$ satisfies the same conditions as in Theorem I. If $x(t)$ is a solution to (1) defined at $t=0$, then $x(t)$ can be extended to $[0, \infty)$ and there is a constant $c$ such that

$$
|x(t)| \leqq c+3 \int_{0}^{t}|h(s)| d s, \quad \text { all } t \geqq 0 .
$$

Proof. Let $[A, B], H(t), M_{A, B}$ and $y(t)$ be as in Lemma II. Suppose $\dot{x}(t)-h(t) \geqq 0$ on $[A, B]$. Just as in Lemma II, if $A \leqq t \leqq B$,

$$
\begin{aligned}
a(t) G\left(y(t)-M_{A, B}\right) \leqq & a(A) G\left(y(A)-M_{A, B}\right) \\
& +\dot{y}(A)^{2} / 2+\int_{A}^{t} \frac{a^{\prime}(s)}{a(s)} a(s) G\left(y(s)-M_{A, B}\right) d s .
\end{aligned}
$$


In this case, Gronwall's inequality implies that $a(B) G\left(y(B)-M_{A, B}\right) \leqq\left[a(A) G\left(y(A)-M_{A, B}\right)+y(A)^{2} / 2\right] a(B) / a(A)$.

From this,

$$
|y(B)| \leqq|y(A)|+\mu+2 M_{A, B}
$$

where

$$
\int_{0}^{\mu} g(s) d s=\left(\dot{y}(A)^{2} / 2\right) a(A)^{-1}, \quad \mu \geqq 0 .
$$

(The same result holds if $\dot{x}(t)-h(t) \leqq 0$ on $[A, B]$.)

Since $|x(B)| \leqq|y(B)|+M_{A, B}$ and $x(A)=y(A)$,

$$
|x(B)| \leqq|x(A)|+\mu+3 M_{A, B} \text {. }
$$

The rest of the proof of Theorem II proceeds much as that of Theorem I and will be omitted.

We note that in both of these theorems, if $h(t)$ is constant, then all solutions are bounded. (See [4].) We conjecture that the boundedness of all solutions to $\ddot{x}+a(t) g(x)=0$ is sufficient to insure that any solution to (1) is $O\left(1+\int_{0}^{t}|h(s)| d s\right)$ as $t$ goes to infinity.

If $a(t)$ is not bounded below by a positive number, then the conclusion of Theorem I does not hold. In fact, if $\int^{\infty} s a(s) d s<\infty$, then there is a solution to $\ddot{x}+a(t) x=0$ which is asymptotic to a straight line of positive slope $[1$, p. 103]. In this case, the following theorem provides a bound in the (very restrictive) case $|g(x)| \leqq k|x|^{\alpha}$ for some constant $k$ and $\alpha \in[0,1]$. We can remove the restrictions on the signs of $a(t)$ and $g(x)$ in this case.

We might comment here on a result in [5] to the effect that if $\int^{\infty} t^{\alpha}|a(t)| d t<\infty$ and $|g(x)| \leqq k|x|^{\alpha}$ for some $k$ and $\alpha \geqq 0$, then all solutions to $\ddot{x}+a(t) g(x)=0$ have bounded derivatives. In fact, this is false unless we restrict $\alpha$ to [0,1]. For example, with $a(t)=-2 / t^{4}$, $g(x)=x^{2}$, the function $x(t)=t^{2}$ is such a solution.

TheOREM III. Suppose $|g(x)| \leqq k|x|^{\alpha}$ for some $k$ and some $\alpha \in[0,1]$. Suppose further that a $(t)$ is continuous for $t \geqq 0$ and $\left(^{*}\right) \int_{0}^{\infty} t|a(t)| d t<\infty$. Let $x(t)$ be any solution to (1) defined at $t=0$. Then $x(t)$ can be extended to $[0, \infty)$, and $x(t)=O\left(t+t\left|\int_{0}^{t} h(s) d s\right|\right)$ as $t$ goes to infinity.

Proof. We can write, as far as $x(t)$ is defined,

$$
x(t)=x(0)+t \dot{x}(0)+\int_{0}^{t} h(s) d s-\int_{0}^{t}(t-s) a(s) g(x(s)) d s,
$$


and if we let $H(t)=\int_{0}^{t} h(s) d s$, then there are numbers $c_{1}$ and $c_{2}$ such that

$$
\begin{aligned}
|x(t)|+1 \leqq & c_{1}+c_{2} t+|H(t)| \\
& +\int_{0}^{t}(t-s) k|a(s)|(|x(s)|+1) d s .
\end{aligned}
$$

Lemma III. Suppose that $\phi(t)$ is an integrable function on $[0, T]$, and for $t$ in this interval,

$$
\phi(t) \leqq A(t)+\int_{0}^{t}(t-s) B(s) \phi(s) d s
$$

where $B(t)$ is continuous and nonnegative and $A(t)$ is continuous. Let $z(t)$ be any solution to $\ddot{z}-B(t) z=0$ which is positive for all $t \geqq 0$. Then for

$$
t \in[0, T], \quad \phi(t) \leqq A(t)+z(t) \int_{0}^{t} \int_{0}^{s} A(u) B(u) z(u) / z(s)^{2} d u d s .
$$

Proof of Lemma III. Let

$$
R(t)=\int_{0}^{t}(t-s) B(s) \phi(s) d s .
$$

Then $\ddot{R}(t)=B(t) \phi(t) \leqq B(t) A(t)+B(t) R(t)$. Let

$$
Q(t)=\int_{0}^{t} B(s) z(s) d s+\dot{z}(0) .
$$

Then we have $d[z(t) \dot{R}(t)-Q(t) R(t)] / d t \leqq B(t) A(t) z(t)$. Integrate, divide by $z(t)$ and multiply by $\exp \left\{-\int_{0}^{t} Q(s) / z(s) d s\right\}$, and we obtain

$$
\begin{aligned}
& \frac{d}{d t}\left(R(t) \exp \left\{-\int_{0}^{t} Q(s) / z(s) d s\right\}\right) \\
& \quad \leqq \exp \left\{-\int_{0}^{t} Q(s) / z(s) d s\right\} \cdot \int_{0}^{t} B(s) A(s) z(s) / z(t) d s .
\end{aligned}
$$

The result follows by integrating again and noting that

$$
Q(t)=\int_{0}^{t} B(s) z(s) d s+\dot{z}(0)=\int_{0}^{t} \ddot{z}(s) d s+\dot{z}(0)=\dot{z}(t) .
$$

Now we note that condition $\left(^{*}\right)$ insures the existence of a solution $z_{1}(t)$ to $\ddot{z}(t)-k|a(t)| z(t)=0$, with $\eta \leqq z_{1}(t) \leqq 1$ for some $\eta>0$, all $t \geqq 0$. 
(See [1, p. 103].) Using Lemma III with (6) gives

$$
\begin{aligned}
|x(t)|+1 \leqq & c_{1}+c_{2} t+|H(t)| \\
& +z_{1}(t) \int_{0}^{t} \frac{1}{z_{1}(s)^{2}} \int_{0}^{s} k|a(u)| z_{1}(u)\left[c_{1}+c_{2} u+|H(u)|\right] d u d s \\
\leqq & c_{1}+c_{2} t+|H(t)| \\
& +\left(k / \eta^{2}\right) \int_{0}^{t}(t-s)|a(s)|\left[c_{1}+c_{2} s+|H(s)|\right] d s .
\end{aligned}
$$

Clearly $x(t)$ can be extended to $[0, \infty)$. For $t>1$,

$$
\begin{aligned}
& \frac{|x(t)|+1}{t+t|H(t)|} \\
& \quad \leqq \frac{c_{1}}{t}+c_{2}+1+\left(k / \eta^{2}\right)\left[\int_{0}^{t} c_{2}|a(s)| s d s+\int_{0}^{t}|a(s)|\left(c_{1}+1\right) d s\right]
\end{aligned}
$$

and the result follows from $\left({ }^{*}\right)$.

\section{BIBLIOGRAPHY}

1. E. A. Coddington and N. Levinson, Theory of ordinary differential equations, McGraw-Hill, New York, 1955.

2. J. E. Potter, Some statistical properties of the motion of a non-linear oscillator driven by white noise, Ph.D. thesis, Massachusetts Institute of Technology, Cambridge, Mass., 1962.

3. E. J. Putzer, The rate of growth of solutions of a second-order differential equation, J. Soc. Indust. Appl. Math. 10 (1962), 454-468.

4. P. Waltman, Some properties of solutions of $u^{\prime \prime}+a(t) f(u)=0$, Monatsh. Math. 67 (1963), 50-54.

5. Bing-ten Zhang (Ping-Ken Chang), Boundedness of solutions of ordinary differential equations of the second-order. Acta Math. Sinica $14(1964)=$ Chinese Math. 5 (1964), 139-148.

Case Institute of Technology 